\title{
Selecting System Development Tools: \\ Some Experiences
}

\author{
REIMA SUOMI \\ LisSc(Econ) \\ Turku School of Economics and Business Administration \\ Sampo Insurance Company Limited
}

\begin{abstract}
Case-tool market is quite unstructured and not mature. It is no wonder that buyers are more than blurred. Several acquisitions have so far been based on false or insufficient data, which has led to lost money and other resources.
\end{abstract}

Some companies have even worsened their application backlogs because of unwise use of wrong case-tools. Experiences from real-life situations should contribute to the understanding of these problems considerably. This article tries in its part to fulfill this gap.

This article describes implications and results of two software selection processes in a Finnish insurance company. First, during year 1987, the company selected an application generator for mainframe use and for mainframe-based systems. During year 1988 this acquisition was followed up by a selection of a tool for system requirement analysis and description purposes (a case-tool) which should be based on PC's.

Both acquisitions were based on a very detailed process, the experiences of which are summarized here. This is done by the means of describing the phases every good selection process should include. For each phase, targeted results are described and reasons to go through the phase are served. 


\section{Introduction}

The need to make systems work more efficient is today more urgent than ever before. Technology is evolving at a fast rate and old systems become outdated faster than ever. Updating and maintenance of old systems is becoming a major burden for most companies ${ }^{1}$. In addition, new, yet unseen, application areas such as artificial intelligence, inter-organizational information systems and new generations of decision support systems are emerging. One study [Martin 1982, referring to a study by IBM] has found out that the number of requests for new applications is increasing at a $45 \%$ yearly rate in enterprises. Another study [Case 1986] by U.S. department of defense found out that system work productivity will grow only $4 \%$ yearly, whereas demand is growing at a pace of $12 \%$ annually. Both new and old applications increase the total burden of system developers:

\section{Figure 1:}

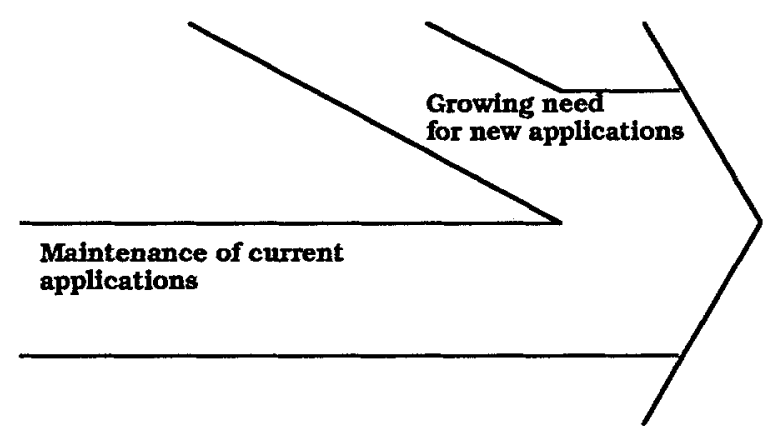

The increasing amount of systems work needed

The industry and organizations have tried to solve this problem by various means in different times. Three distinctive periods seem to emerge:

$$
\begin{array}{ll}
1 & \text { development of methods } \\
2 & \text { development of organization } \\
3 & \text { development of tools }
\end{array}
$$

1 Common pitfalls in information system planning are summarized in a study by Lederer \& Sethi [1989] 
In the first instance, different kinds of techniques, methods and methodologies were developed in order to intensify and lead system work. Structured techniques, life cycle -oriented thinking and project organizations were children of this time. The main invasion of these techniques was in the beginning of the 1970's. The nature of the problems - they were mainly technical - allowed this kind of approach quite well.

During the late 1970 's, winds of distribution and decentralization began to emerge. It was understood that the nature of the system development problems was mainly social and organizational, and the end users were tied to the processes. Information system development resources were assigned to the management of the basic business functions.

In the 1980's - after the invention of microcomputers - focus has turned to the different tools available for system development. Microcomputers were the needed technology which allowed cost-efficient and userfriendly tools to emerge to the tables of system developers as well as end users. This means that the methods have already established themselves - at least some of them - and that distribution is seen as an established method of organizing information technology and its development resources. New efficiency is sought from tools more eagerly than ever. According to a study by McClure [1988], the total market for front-end tools (case-tools) is up to 500000 copies, for code generators at least 7000 . By 1987, a modest 2 percent penetration level was accomplished.

The classification of different tools for development of systems work would be an endless topic, here we will suffice ourselves with the view that these tools can be divided according to three dimensions:

1 front end tools - back end tools -front end tools are for logical development of the system, back-end tools for technical development

2 tools for professionals - tools for end-users

3 tools for individual use - tools for group use 


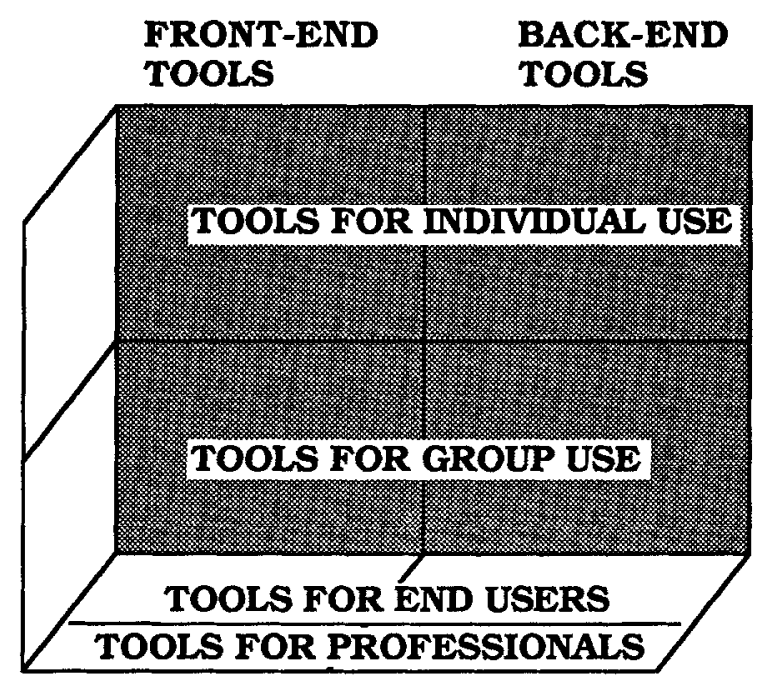

Classification of system development tools

System development - especially the phase of coding - has always been a manual, work intensive and error-prone phase of system development, which has allowed few possibilities for making the work more efficient.

It has long been known that the elimination of these manual work phases would be the key to more efficient systems work. The most visible drawbacks of manual operations are:

- the unbroken chain is spoiled: changes in one or other end are no more automatically transferred to the other end

- the manual operations easily produce errors

- yet only a small part of the total work is manual, the human worker must introduce her/himself to the whole chain of actions - the manual operations take time

The current automated techniques of software engineering and automated tools did not anyway occur overnight, but needed time to mature and lay on three basic innovations or approaches in system development methodologies that have occurred during years: 


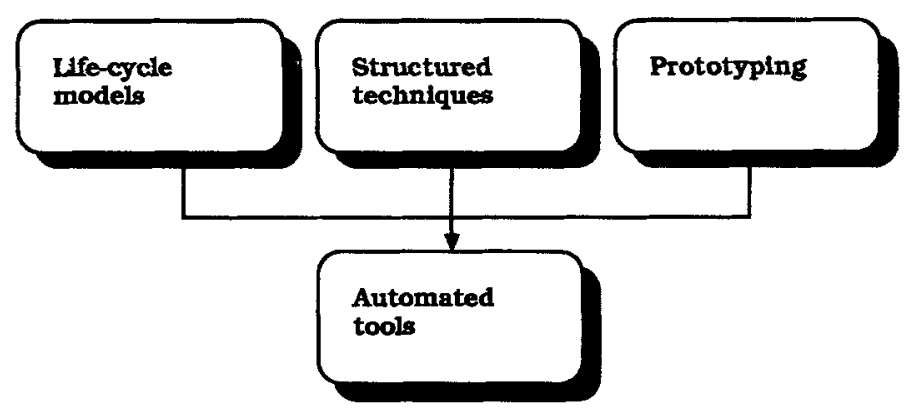

Approaches leading to automated tools

Life-cycle models laid the major theoretical but also practical view on how system development should be divided into phases. The major differentiation between logical and technical planning, implementation and maintenance is still valid today. The phases of coding and testing are beginning to fade away as new automated tools take hand of these phases.

Structured techniques provided the major tools for describing complex systems in quite a simple way. These techniques, however, need a lot of drawing and updating, a work that has become tolerable first now as we have new tools to be used. The future importance of the structured techniques is stressed for example in books by Martin \& McClure [1985] and in an article by Yourdon [1986].

Prototyping approach brought in the innovation that systems should be made visible already when they are still under development. This is just the way new system development tools work: they allow to view not finished systems and make improvements and changes easy to make.

Nowadays it is also possible to get at least very near to the final mode: the users give their logical insight on how the system should work, and different automated tools will build up the working information system based on these logical descriptions: 
Figure 4:

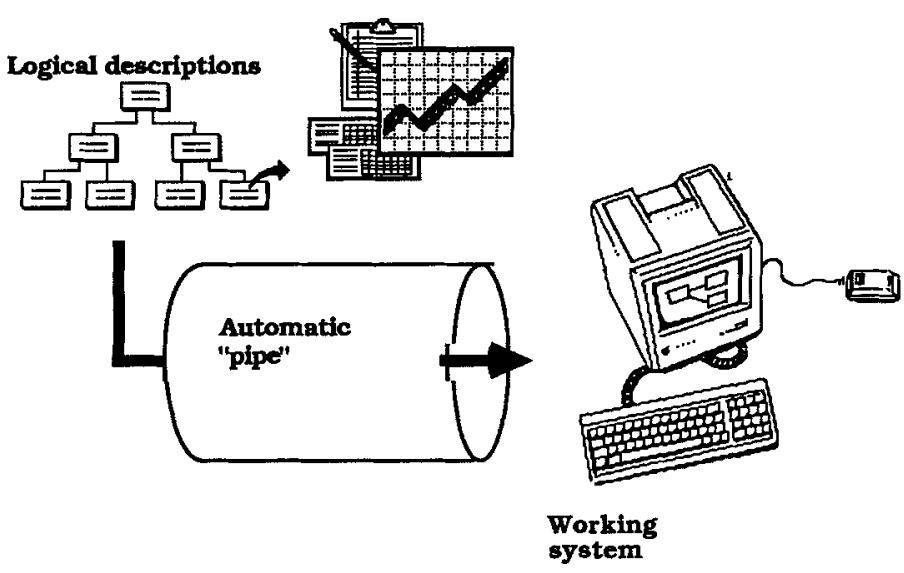

The ideal of systems work.

\section{Methods: the tailoring and customer approaches}

There seems to be two main approaches (in addition to the option of making all the needed programs self, which is hardly a cost-efficient method for most companies) for acquiring automated support for system development.

First, it is possible to buy a tool that covers up the whole lifecycle. Such products are usually closely tied to some particular methodology, and include all the major parts of a case tool: support for all the phases of the system work from strategic system portfolio planning to the maintenance of individual systems. The buyer of the tool enters a very close customership to the vendor, so the name customer approach.

Second, it is possible to buy a set of tools, each of which is especially designed to support one or a few of the phases of system development work. In such a way a set of state-of the art products can be selected, but the integration between the tools may be less than optimal. This approach necessitates a lot of tailoring work, so this name. 
Within the tailoring approach, products in very many price categories can be found. In general, the case market seems to be in a play of decimals. There are three classes from which to select the tools:

1 full packages

- include tools for analysis and design, implementation plus a method framework

- price about 1000000 FIM

2 normal packages

- include tools for analysis and design

- price about 100000 FIM

3 modular packages

- include tools for some phases in analysis and design

- price about 10000 FIM

The differences of the tailoring and customer approaches from some critical viewpoints are discussed here:

The critical success factor for individual tools is their connectibility. Nearly all these tools have options for data input and output, and actually a link can always be programmed between two tools with quite a bearable effort. The trouble sets in when new versions of the products are introduced, and the tailored links must be programmed every time anew.

What is needed is different standards that link these products together. The trend seems to go to the same situation as is seen in other fields of information technology: there are official standards such as EDIF or IRDS, and then there are practical standards set up by dictionary/repository manufacturers. In this field, time will show which will be the effect of the new repository of IBM.

The organization which is to use these products must take into attention it current investments. If one can set up from an empty table, a total new system according to the customer approach may be the best solution. The trouble is just how to find the right product. When a total commitment can be foreseen, it should be to a product with future. No matter how small part of the total system development work the new tools covers, if links are to be built to other tools and work is to be based on this tool, future support from the vendor of the tool will most surely be needed. In addition to the vendor local importors and distributors must also be engaged to the product. 
When current systems are highly usable and will surely need support also in the future, the tailored approach may be of use. When tailoring links between the system development software packages, links to current production systems can also be considered. The tailoring approach of stepwise nature also leaves room for slow replacement of current systems.

From the viewpoint of the required new investments, the tailoring approach seems to be easier to suffer: quite low initial investments will suffice. On the long run, the cost will anyway rocket. New software will be constantly needed (every workstation will usually require its own license), and the tailoring work going on in the organization can become a major burden. In addition, the ongoing education in small groups and during work can lower productivity more than a one-time total commitment to the education effort. The customer organization will usually have to tailor its own methods, and this will most surely also consume a lot of resources. The customer approach at its side usually means a million-investment right from the beginning, but with the sum usually an unlimited number of workstations is achieved (the programs supporting the whole life cycle usually reside on mainframes) and the method and education support is settled at the same time.

The customer approach usually means big influence from the side of the vendor. They tend to offer support, methodologies and consulting with the actual software package. The costs of these services very easily nearly double the price of the solution, if they are not included in the total package. In general, the costs of introducing a new tool include at least following parts:

- $\quad$ build-up of links to other tools

- build-up of links to system software

- build-up of necessary standards

- $\quad$ training of personnel on the use of the new product

- $\quad$ salaries of the personnel responsible for the product

- maintenance costs of the product.

In general, the acquirement of the tool should be based on mutual confidence. If too much pressure is applied from the side of the customer to the price or other terms of the agreement, this will produce later lower service levels. If the seller gets the price it has calculated will cover the expenses caused by the service, it will also be willing to give that service. Bargaining is always needed, but must not be 
practised into extremes. It must be remembered, that the price paid to the vendor is only a small part of the total expense, and good relations to the vendor can clearly lower other expenses.

What must be considered is own experience. If new methods of system work are really needed and investments in them seem anyway unavoidable, the customer approach with the acquirement of a complete methodology is reasoned. But if own methods and ways of doing things are well established and of state-of-the-art quality, there is no reason to abandon that investment and make a new. The tailoring approach with moderate investments seems to be a wiser approach.

The totality of the customer approach is the risk of this approach. Risk is here a way of assessing things, not absolutely a bad thing. With the customer approach, the results can be either very bad or very good ones. With the tailoring approach, risks are lower (investment is lower), but the possible outcomes are too only at a moderate level.

The differences between the two approaches are summed up in table 1:

Table 1:

Tailoring approach

Best tools for every phase
of system work
Methods can be tailored

Risk usually manageable

Lot of tailoring work needed

Integration with current

systems easier to achieve

Can be built up in phases

Investment in small doses, total investment may be bigger

Learning by doing

Commitment can vary

Suitable when current applications portfolio big
Customer approach

Best support for total

life cycle support

Usually tightly tied

to one method

Big risk

Usually little tailoring work

Integration with current systems

difficult to achieve

Taken into use in one effort

Big one-time investment, total investment maybe smaller

Learning by vendor education

Total commitment needed

Suitable when current applications

portfolio not big

Differences between the customer and tailoring approaches 
The size of current applications portfolio must be taken into attention when the desired approach is selected. The introduction of a new comprehensive toolset tends to lead to a situation where the new applications and the old ones are very loosely coupled. The process of the introduction of the new tool leads to a situation where new systems are built with the new tool, but where very little support is given to the maintenance of the old application portfolio, which originally was the problem. So, an environment with established tools and applications and a lot of maintenance work seems to necessitate the tailoring approach. If there are only few old applications, a totally new generation of applications and methods is easier to begin.

\section{A stepwise approach to selecting software products}

Published comments and descriptions on software selection processes leave behind a picture of a quite random process ${ }^{2}$. Many times not even the minimum trouble of a decent selection process is met, but the decision on software products to be used is based on some other factors such as:

- $\quad$ one product, usually the one that seems to be the market leader, is picked up randomly

- the product that is most eagerly marketed is selected

- the product with the lowest start-up price is selected

- a product sold or recommended by the hardware vendor or some major software vendor is selected

- the personal relationships or attitudes of higher management dictate the product to be selected

- the product that a competitor or mother or daughter company is using is adopted

These approaches however many times seem to result in failures. Reasons for failures stem from two sources: either from inside or from outside of the company selecting the software. The main reasons for outside reasons are the failures and shortcomings in the products, but

2 This in spite of the fact that there are many detailed descriptions how on how software tools should be selected [Gibson 1989, Lucas. \& al 1988, Martin 1985, McClure 1988] 
they are left unnoticed by the improper selection processes inside the company. These are caused by false assumptions and behaviors.

The usual mistakes made are summarized in table 2:

Table 2:

-too cumbersome and laborious use of the product -mistakes and flaws in the product

-bad support for the product

-unplanned need for acquisitions

-bad reception of the selected product

-incompatibility with other software

-too heavy use of machine resources

Usual mistakes during a software selection process

During the selection process ideas and opinions about the tools to be selected have time to mature. In order to make the selection a real group work which produces confidence and engagement to the new product, the group responsible for the selection should have members from all the major interest groups of the new tool, as described in the figure:

\section{Figure 5:}

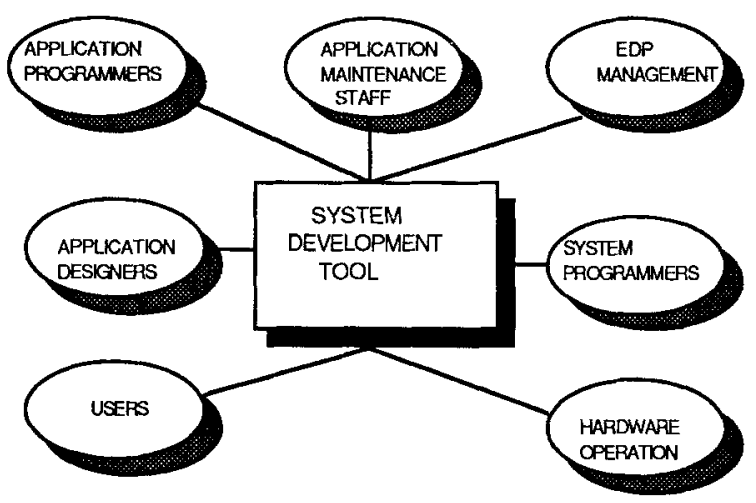

Interest groups of a system development tool 
The time needed for the selection process should obviously depend on the planned useful life of the product to be acquired. If you are selecting a tool to be used for some months or perhaps a year, not much time can be spent on the selection. On the other hand, if you are selecting the backbone of your application development to be used for decades, a year seems a short time.

The author was responsible for selecting two major productivity tools for system development to one of Finland's biggest insurance companies. With the goal of automated system life cycle, the process was divided into two phases: first a back-end tool for code generation was selected, since this was the phase of most urgent need for help, and a year later a front-end tool to be joined in front of the back-end tool was selected ${ }^{3}$. The reasons for first selecting the back-end tool were:

- $\quad$ programming is nearest to maintenance, the area of biggest problems. It was hoped for that by automating code generation maintenance could be automated little by little.

- $\quad$ programming seems to be a quite structured task which could maybe be automated quite easily

- good programmers begin to be a scarce resource

- the tools used here were quite oldfashioned in the company (conventional third generation languages)

- testing of technical details is a time-consuming task, which can be eliminated by automated code generation

During the whole process it was clear that the tools to be selected would be for edp-professionals ${ }^{4}$ only. The heavy transaction-processing applications of an insurance company are of such character that professionals will anyway have to do the final work.

The main idea was too that system development is a group work, and so tools allowing for group work, in practice tools with network capabilities and common data-bases, should be selected.

According to our experiences that were collected during those projects, at least the following phases should be carried out when a productivity tool for system development is selected:

3 This kind of stepwise approach will most clearly lead to the tailored approach, as was the output here

4 This is not to say that there were no projects to help end-users in the company, only one of the many edp-projects in the company is described here 
Figure 6:

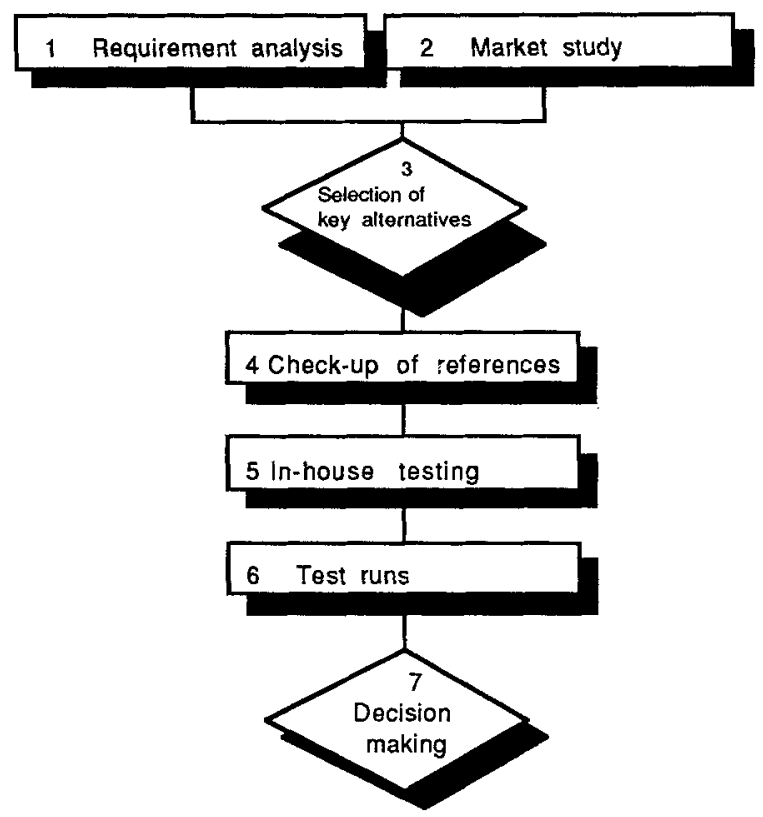

Phases of a proper software selection process

First a proper requirement analysis is needed. Goals stated in a general way are not enough: "efficiency for development work", "good user interface", "connections to application generator" are not detailed enough requirements. What is needed is a set of requirements that serve as a basis for concrete questions that can be answered "yes" or "no". An example of a requirement might be "an user interface with windows" and the concrete questions like "can at least three windows be open at the same time?", "can the size of the windows be changed?" and "is it possible to cut and paste graphical objects between the windows?".

The requirement analysis should be done with a wide group of people. As many opinions as possible should be acquired. What should be first is the need of the interest groups of the system, not for example the needs of the current hardware architecture or repository. Here, as in other fields of applications, we should focus first on the needs of the users and customers of the new tools, then we should select the best of the available technology bases for fulfilling this need, and first the third task should be the decision on the product. 


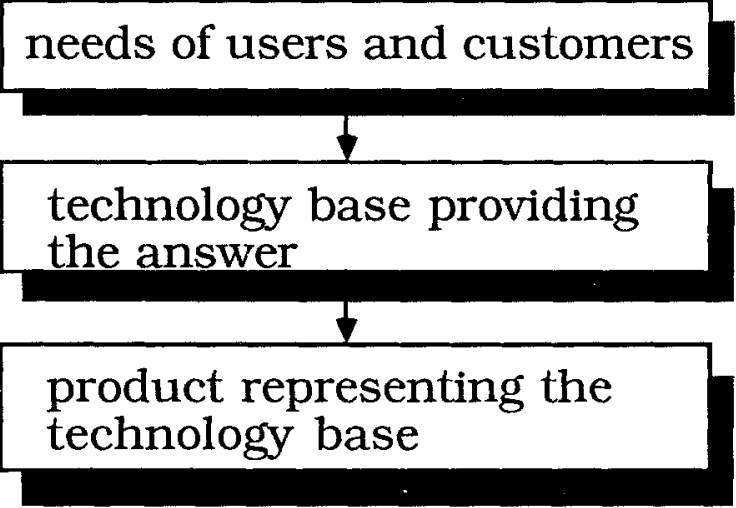

Where to focus on which order

A market study is especially necessary with markets that are very dynamic like the current case-tool -market where knowledge on the market gets old pretty soon. A proper market study collects information on at least the following factors:

- products available (name, price, reductions, domestic users and users abroad (number of licenses and number of customers)

- distributors of the products (service available, market share, resources, other products distributed)

- vendors of the products (service available, market share, resources, other products supplied).

Data during the market study phase can and should be collected from journals, newspapers, product reviews, professional meetings, seminars, prospects of the vendors - and from hearsay too, which can be a very valuable source of knowledge.

Sooner or later the buyer will have to make a selection of key alternatives. The phases of the process from this point on tolerate only a limited number of alternatives, usually from two to four. Usually the key alternatives represent different technologies, and the best product representing each technology should be taken into further considerations. 
Visits to reference companies are many times the most illuminating part of the process. What must be remembered is that they should be based on mutual trust and change of information, i.e. you will have to tell about your own intentions and results of the market study, too. In general, it must be remembered that companies that have acquired some products tend to stay behind them also when they are not quite satisfied. So, don't let the discussion go on in a general level in the reference company, but try to get into the daily operations level. Ask, how is this and that task done, not how well the tool supports work in general.

In-house testing of the products is always needed. For example, current case-tools include thousands of details and it is impossible to get an impression on them based on demonstrations and short viewing. Usually the time to learn the usage of a case-tool is from one week to one month. First after this threshold it is possible to begin to do efficient work, work which first reveals the virtues of the product.

Case-tools can be best tested by building anew some old applications with the new tool. If you try to do too much at a time and design a completely new application you may lose your strength to the application, not to the test itself, which should be the most important thing in the selection process.

In-house testing gives some quidance about the user interface, the working methods with the tool, and similar technical aspects, but conclusions on the impact of the tool on the total productivity of the company can not be derived. System development tools mature first after they are properly istalled to the company, a process which can take years.

Test runs in own machines are needed in order to find out how much resources the software to be acquired needs. The resources to be looked after are consumption of CPU-time, input-output capacity and central memory. All are relevant in mainframes, central memory sizes can be very critical in micros. Exact figures - not to talk about comparisons between different products - are not available from vendors. Usage of resources should be tested in two different points, when

- the product is used for building applications

- the applications built with the products are used. 
Decision making should be based on the results of all the phases of the selection process. Who are the decision makers depends on the organization, but usually they should be the managers who will be responsible for the future application of the tool and who must pay for it. Special care should be taken to beware strong managers who try to bypass the selection process and justify their own personal but unproven opinions.

Usually there is a tendency to give bigger value to the results gained most recently. Anyway, if all the other phases of the work have indicated results that are not in line with the results of the in-house tests, these results should also be taken into attention when making the final decision. On the other hand, when the process described here is followed, no bad products will ever come to the testing phase.

During the whole selection process, a lot of reports should be produced and circulated effectively. The key activity is to engage the whole organization into a discussion about the alternatives. This can only be gained by literal documents in an organization bigger than ten employees, and the application of literal material also gives the process the needed openness and rigidity. Special care should be taken when the decisions are documented: on which data they were based and who made them.

\section{Discussion}

Productivity tools for software development are clearly needed. In addition, they are a clear product of logical developments in the field of system development. Organizations should familiarize themselves with these tools and try to use them in the best possible way. But there is no reason to do unargumented decisions as seems to be the trend today. By taking into attention some general and simple rules as presented here the organizations will be much better off.

Productivity tools for system development are a comparatively new phenomenon, and there is no reason to marvel at why the market is in such an unmature state as it is. In such a kind of market, possibilities of wrong actions are very high. Some of the major characteristics of the current markets seem to be: 
- most companies have selected their tools in a random way, usually based on personal preferences of some managers

- the ideal of an automated life-cycle is still far from fulfilled: current tools are mainly used to support distinctive phases of the system work, integrating links are a rarely met

- a parade application seem to be analysis of conceptual modelling for data-bases ${ }^{5}$, many companies have not at all used the tools for process planning

- the tools are difficult to learn, getting familiar with the structured methods involved is a hard task (as expected)

- some 30-40 back-end tools which necessitate a deeper commitment have been sold in Finnish market, front-end tools are acquired mainly for testing purposes in low quantities

- $\quad$ results from many pilot projects have been unsatisfactory, many products have been abandoned

the main advantage of new system development tools seems to be that of improved quality, not that of efficiency

some companies, however, had acquired considerable short-term gains in the form of saved work

- the tools remain islands of technology, no links to other software tools of organizations have been built

- no adjustments of system development methods has occurred because of the tools 6

The whole situation of the market should become better if software buyers were more critical and capable of conducting thorough selection processes. So the whole market would be under a harder pressure and the best products would be selected from the mass sooner. Currently, the unmature state of the productivity tool market is inhibiting development.

The last advice is: begin work on system development tools such as application generators and case tools today. By beginning is meant interest on the issue, not a selection of a comprehensive tool right away. Usage of system development tools is not a metro train that passes by daily and to which you can jump whenever you want. It is hard work, and if you want results in a few years, you must take the drivers seat on the metro right now.

5 This fact is for example recognized by Yourdon who is to be considered one of the "Fathers" of structured techniques [Yourdon 1988].

6 This is considered to be one of the major failures of case-tool usage by Inmon [1987] 
References:

Case, Albert F., Jr. (1986) Information systems development: principles of computer-aided software engineering. Prentice-Hall.

Gibson, Lucas Michael (1989) Implementing the promise. Datamation February 1./89, 65-67.

Inmon, Peter (1987) CASE no cure-all. Computerworld November 23./87.

Ledered, Albert - Sethi, Vijay (1989) Pitfalls in Planning. Datamation June 1./89, 59-62.

Gibson, Lucas Michael (1989) Implementing the promise. Datamation February 1.2/89, 65-67.

Lucas, Henry - Walton, Eric - Gingberg, Michael (1988) Implementing Packaged Software. MIS Quarterly December/88, 537-549.

Martin, James (1982) Application Development without Programmers. New Jersey.

Martin, James - McClure, Carma (1985) Diagramming Techniques for Analyst and Programmers. New Jersey.

Martin, James (1985) Fourth Generation Languages, Volume I, Principles. New Jersey.

McClure, Carma (1988) The CASE technical report. Extended Intelligence, Inc. Chicago.

Yourdon, Edward (1988) Case competition is all over the world. Software Magazine November/88, 53-60.

Yourdon, Edward (1986) What ever happened to structured analysis? Datamation June/86, 133-138. 